\title{
Inspired to improve outcomes in pancreatic cancer
}

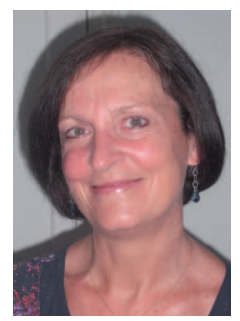

Pippa Corrie speaks to Natasha Galukande, Assistant Commissioning Editor. Dr Corrie undertook a PhD in anticancer drug development before qualifying in medicine at Oxford University (Oxford, UK) and training in medical oncology at the University of Birmingham (Birmingham, UK). Pippa is now a Consultant Medical Oncologist and University Associate Lecturer at Cambridge University Hospitals NHS Foundation Trust (Cambridge, UK). She is Clinical Director for Systemic Therapies in the Cancer Division at Addenbrooke's Hospital (Cambridge, UK), which sees over 3000 new patients each year, and lead Oncologist for the pancreatic cancer service at Addenbrooke's Hospital and across the Anglia Cancer Network. She is Deputy Director of the Cambridge Cancer Trials Centre (Cambridge, UK) and a member of the National Cancer Research Institute Pancreatic Cancer Clinical Studies Group. Her goal is to improve outcomes for patients with chemoresistant cancers through the conduct of clinical trials. Her specialist interests are pancreaticobiliary cancers and melanoma, researching mechanisms of response and resistance to anticancer drugs.

\section{What sparked your interest in pancreatic cancer?}

As a junior research fellow in the 1990s I worked in the gastrointestinal cancers clinic at a time when there was no standard of care for pancreatic cancer patients who had an extremely short life expectancy. In 1997 the Burris paper was published reporting a survival benefit from gemcitabine (Gem) compared with 5-fluorouracil [1]. It caused quite a stir internationally, but most UK clinicians were quite cynical at the time regarding the small survival benefits that the study showed despite the fact that they used a clinical benefit response, which at the time was quite innovative. However, I was convinced this was a first step in the door for such a needy patient group and worked closely with colleagues in Lilly (Basingstoke, UK) to help prepare their case to NICE, which ultimately led to positive guidance in 2001. I have worked to improve outcomes in this disease ever since.

\section{- How did you become involved in clinical trials for improving the treatment of pancreatic cancer patients?}

I was interested in cancer and its treatment before I qualified as a doctor. I completed an intercalated $\mathrm{PhD}$ in cancer research aimed at understanding the mechanism of action of second-generation platinum agents being developed at the time, which stimulated my curiosity in drug development at a preclinical level. Later on, as a junior doctor, I found the evaluation of new treatments in the clinical setting just as exciting.

As a research fellow I was working in Birmingham as the oncology contact for a surgeon, John Neoptolemos (University of Liverpool, Liverpool, UK), who also had a passion for pancreatic cancer and, knowing how poor outcomes were after surgical removal, had set up a randomized trial to evaluate adjuvant therapy in patients after Whipple resection. John set up the first ESPAC trial with no funding support; his sheer determination made it happen. He would give me a call when he had a patient in the clinic who needed consenting and, if randomized to treatment, it was my job to set it up. It was also an eye opener for me regarding informed consent at a time well before the EU Clinical Trials Directive - since many of the patients around Birmingham were of Asian origin and unable to speak English, information was usually conveyed via a child acting as interpreter so informed consent was sometimes rudimentary. My eyes were opened to how clinical trials were conducted. I was also very impressed that this surgeon had made the effort to run this trial because he was so concerned. That was the first of a series of ESPAC trials that established the role of adjuvant therapy in this disease. I think that first experience really inspired me to continue in clinical trials.

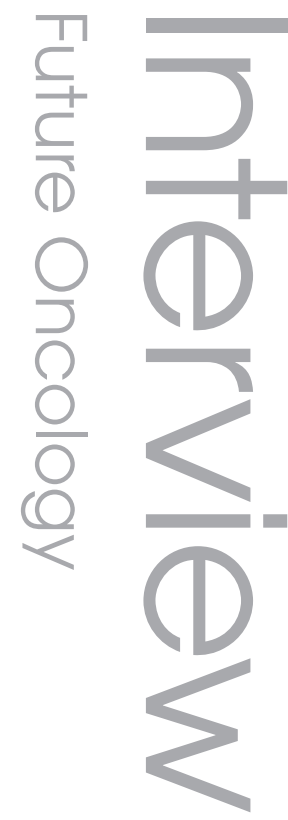

Future $\%$
Medicine $\%$ part of 
- Can you describe one of your first key experiences with cancer patients?

As a junior doctor in general medicine I was on call one night and bumped into an oncology registrar at midnight teaching junior doctors how to prescribe cisplatin hydration. I was quite shocked at the dedication and the madness of this registrar. I then came to realize that in oncology you do have to be very dedicated and motivated. I think it reflected the fact that people in this field work very hard and go the extra mile for their patients.

\section{- What do you feel is the biggest challenge in the field today?}

There are many challenges but, in my view for pancreatic cancer specifically, the biggest is the predicament we have in diagnosing this condition early enough. At the moment under 20\% of patients with pancreatic cancer present with operable disease and even these patients have an extremely poor outlook: at best, only $20 \%$ will survive 5 years, despite potentially curative surgery. Although there are many people like me interested in drug development, really we are only the icing on the cake and to make a difference we need to be improving our surgical rates because that is the only chance of cure. The problem is that there are lots of barriers to early diagnosis of this disease, some cultural, some about the disease itself - signs and symptoms preceding diagnosis are often vague and nonspecific. I would like us to find a test or imaging modality that enables us to detect disease early that could be ultimately used in population screening.

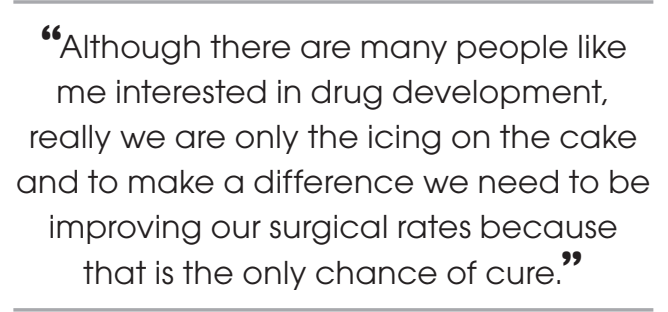

We do need to raise awareness of this devastating disease. In our society, GPs tend to be the gatekeepers of healthcare. Our systems generate barriers to care rather than facilitators. Most people do not like to see doctors at the best of times and real-life difficulties in accessing even a simple GP appointment means that people are even less inclined to seek help even when they really need it. I suspect these kind of cultural habits contribute to poor disease outcomes in our country and speak of a need to change perceptions regarding the role and remit of our healthcare professionals.

\section{- As a chair of the National Cancer Research Institute melanoma clinical studies group \& a member of the National Cancer Research Institute pancreatic cancer subgroup, could you tell our readers what these two roles entail? How do you balance your time between your roles?}

The National Cancer Research Institute has a series of clinical studies groups (CSGs), which are a multidisciplinary group of national leaders in their field whose main role is to develop the research strategy for a particular type of cancer. So, there is a CSG for melanoma and a CSG for upper gastrointestinal cancers. The upper gastrointestinal CSG has a pancreatic cancer subgroup. These groups play a key role in developing national academic trials in their disease area. The group members often lead these trials and work with colleagues around the country to ensure they engage in and recruit to the research studies. We also work very closely with the National Cancer Research Network, whose role is to primarily deliver recruitment to the NIHR portfolio of studies.

Working between the two CSGs is interesting; the groups are very different, they have different dynamics, but the diseases themselves have some commonalities, they are both traditionally chemoresistant, few effective nonsurgical treatments currently exist and patients with advanced disease have poor survival. In metastatic melanoma, in the last 2 years, new treatments have suddenly begun to make a difference, primarily through understanding the molecular basis of the disease. The new treatment modalities in melanoma are immunotherapy and small molecules targeting the BRAF signaling pathway. The reason why the latter has been so impressive is that, through basic science, it became evident that approximately $50 \%$ of melanomas have a mutation in the $B R A F$ gene. Chemists specifically designed a drug to target the active site in mutant BRAF. When given to patients, over $90 \%$ will receive some degree of clinical benefit.

I am hopeful that the step change occurring in melanoma management will inform our approach to pancreatic cancer drug development in the not too distant future.

\section{- What research are you currently involved in?}

I am really a clinical trialist and work as the clinical interface with more basic scientists. 
In melanoma, I am chief investigator for the national adjuvant trial evaluating the role of bevacizumab in patients at high risk of recurrence (AVAST-M). It has completed recruitment of 1343 patients across 53 UK sites and the study is due to report its first interim results at the American Society of Clinical Oncology Annual Meeting this year. Mark Middleton (Oxford University, Oxford, UK) leads the translational component of AVAST-M and my key clinical co-investigators on this Cancer Research UK grant are Paul Lorigan (University of Manchester, Manchester, UK) and Martin Gore (Royal Marsden Hospital, London, UK). We have accumulated an extensive tissue and blood collection associated with the clinical trial, based in Oxford, which is now a major national resource for melanoma research in the UK.

In advanced disease I am working with Ultan McDermott (Cancer Research UK Clinician Scientist, the Wellcome Sanger Institute, Cambridge, UK) to understand mechanisms of resistance to BRAF-targeted therapies. Although there is a high rate of response to such drugs, inevitably virtually all patients become resistant within 6-12 months. Our goal is to genetically sequence tissue taken from patients prior to treatment and on relapse, and identify the mechanisms of resistance, as well as potential therapeutic strategies to offer these patients on relapse.

In pancreatic cancer, we had the honor of Dave Tuveson working in Cambridge and in our team for the last 5 years. During this time, his laboratory was testing new agents in his pancreatic cancer mouse model. They demonstrated that nab-paclitaxel (nabP) was active against this disease, both as a single agent and in combination with Gem. However, in contrast to the recent MPACT trial when nabP was given on the same day as Gem, in the mouse, it appears that greater cytotoxicity was generated if nabP was given $24 \mathrm{~h}$ in advance of Gem. Following release of the MPACT study results, we are planning an exploratory clinical trial to compare concomitant drug administration with sequential administration. By collecting patient tissue and blood samples, the trial will enable us to test whether increased cytotoxicity is due to an impact on cytadine deaminase activity or to the stroma, as originally reported by the Von Hoff group [2]. We hope to secure funding for this study in the next few months.

Working with Dave Tuveson was a great privilege. He came from the USA in 2008 and was the key contributor to setting up our Pancreatic Research Centre in Cambridge. He has now returned to the USA but he has a left a legacy for us. Working with my colleagues including Duncan Jodrell (University of Cambridge, Cambridge, UK), Bristi Basu (University of Cambridge) and Raaj Praseedom (Cambridge University Hospitals NHS Foundation trust, Cambridge, UK), our goal is to maintain our high profile as a major pancreatic cancer center, focusing on novel therapeutics and biomarkers for this disease.

\section{- What are the current 'hot topics' in pancreatic cancer research?}

There are a number of hot topics to mention. First, things are changing in terms of standard chemotherapy for advanced disease. Now we have potential alternatives to Gem, combination chemotherapy both with fluorouracil, irinotecan and oxaliplatin (FOLFIRINOX) and the new nabP/GEM combination regimens are likely to become the standard of care for fit patients. We will need to work out how to sequence these treatments and to also consider what we can offer patients who are not fit for these treatments, as these combination regimens are more toxic and unlikely to be tolerated by less fit patients who make up the majority of those diagnosed with metastatic disease.

"In any consultation with a patient,
whether it is breaking bad news or
discussing entry into a clinical trial of a
new treatment, clinicians need do their
job with humbleness, gentleness and
kindness."

The next task will be to move these combination regimens earlier on in the patient pathway. We need to explore if they can be used in the perioperative setting, or potentially to downstage locally advanced disease to make it operable, which will then impact on the all important surgical resection rates. So-called neoadjuvant chemotherapy and/or chemoradiotherapy are likely to offer the best chance of improving survival outcomes after surgery.

Another key topic, I think, is that, while in melanoma we have a success story in targeting BRAF, in pancreatic cancer $95 \%$ of tumors have a KRAS mutation, but so far we have not been able to effectively target RAS. This is a big conundrum that continues to be the focus of much research in this disease. 
- Finally, what progress do you think will have been made in pancreatic cancer treatment in 10 years time?

I hope that RAS will become a druggable target, which will potentially open up a new era for treating this pancreatic cancer and may even kick cytotoxic chemotherapy into the long grass. This I hope will surely become a reality 10 years from now.

I also hope that we will find technologies and mechanisms for earlier diagnosis. Novel imaging and biomarker technologies being explored, such as circulating tumor DNA, may well provide the answers here. We shall see.

\section{- What has been the proudest moment in your career?}

I do not think I have had my proudest moment as a doctor yet; I have a sense that this is still round the corner for me. What I am most proud of is combining my job with having four beautiful daughters and that is thanks to a great husband who has allowed me to pursue the career I love alongside raising a family.

\section{- What, for you, is the best philosophy in patient care?}

The words that come to mind are always to be humble, gentle and kind. In any consultation with a patient, whether it is breaking bad news or discussing entry into a clinical trial of a new treatment, clinicians need do their job with humbleness, gentleness and kindness.

\section{Disclaimer}

The opinions expressed in this interview are those of the interviewee and do not necessarily reflect the views of Future Medicine Ltd.

\section{Financial \& competing interests disclosure}

$P$ Corrie has no relevant affiliations or financial involvement with any organization or entity with a financial interest in or financial conflict with the subject matter or materials discussed in the manuscript. This includes employment, consultancies, honoraria, stock ownership or options, expert testimony, grants or patents received or pending, or royalties.

No writing assistance was utilized in the production of this manuscript.

\section{References}

1. Burris HA, Moore MJ, Andersen J et al. Improvements in survival and clinical benefit with gemcitabine as first-line therapy for patients with advanced pancreas cancer: a randomized trial. J. Clin. Oncol. 15, 2403-2413 (1997).

2. Von Hoff DD, Ramanathan RK, Borad MJ et al. Gemctibine plus nab-paclitaxel is an active regimen in patients with advanced pancreatic cancer: a Phase I/II trial. J. Clin. Oncol. 36, 5742 (2011). 